\title{
Influence of strict sperm morphology on the results of classic in vitro fertilization
}

\author{
Milton Ghirelli-Filho, Françoise Elia Mizrahi, Antonio Carlos Lima Pompeo, Sidney Glina \\ Projeto Alfa Clinic, Sao Paulo and Faculdade de Medicina do ABC, Santo Andre, SP, Brazil
}

\section{ABSTRACT}

Objective: To determine the influence of the concentration of oval spermatozoa according to the strict morphology criterion in men with normal sperm concentration following the World Health Organization criteria on the results of classic IVF.

Materials and Methods: Based on review of patient charts, this study included infertile couples presenting with female causes for infertility or unexplained infertility, in whom men presented with normal spermogram values for sperm concentration, sperm motility, volume of ejaculate and total sperm count after semen processing greater than 20 million. Based on the value obtained in strict sperm morphology, patients were divided into three groups: in Group A, patients with values between $0 \%$ and $4 \%$; in group B, between $5 \%$ and $14 \%$, and in group $C$, patients with sperm morphology greater than 14\%. The outcomes analyzed were oocyte fertilization rate, biochemical pregnancy rate, clinical pregnancy rate and rate of liveborns.

Results: A total of 244 cases met the inclusion criteria, 27 of them in group A, 165 in group B, and 52 in group $\mathrm{C}$. The mean fertilization rate and the rate of liveborns were, respectively: $71.9 \%$ and 33.3\% in group A; 80.9\% and 24.2\% in group B, and $78.8 \%$ and $28.8 \%$ in group C. There was no statistical difference among the groups in any of the outcomes analyzed.

Conclusion: The values of strict sperm morphology, as proposed by Kruger and adopted by the World Health Organization, had no influence on the results of classic in vitro fertilization in the studied sample.

\section{ARTICLE INFO}

\section{Key words:}

Fertilization; In Vitro; Infertility; Spermatozoa

Int Braz J Urol. 2012; 38: 519-28

Submitted for publication: February, 02, 2012

Accepted after revision: May, 04, 2012

\section{INTRODUCTION}

In 2002, there were approximately 60 million women at reproductive age in the United States; about 1.2 million, or $2 \%$ of these women made a medical visit related to infertility during that year and roughly 10\% underwent some type of treatment for infertility at some time of their lives. Additionally, 7\% of the couples with the woman at reproductive age (2.1 million couples) reported not having reached pregnancy after 12 months of frequent sexual intercourse with no use of contraceptive methods (1).
Among the techniques of assisted reproduction currently available for treatment of these couples, in vitro fertilization (IVF) is the one that offers the best pregnancy rates $(1,2)$. This technique has been used successfully since the late 1970s, and its results show a significant improvement with experience, nevertheless patients with severe spermogram alterations still had little alternative, since the results of IVF for these cases continued poor.

The development of gamete micromanipulation techniques with subsequent intracytoplasmatic sperm injection (ICSI), first performed 
in Brussels, in 1992 (3), brought new horizons to the field of human reproduction and allowed men with minimal sperm production to produce pregnancies, even through alternative techniques for sperm recovery, such as epididymal puncture and testicular biopsy, decreasing the impact of seminal parameters on IVF results.

Unfortunately, not all assisted reproduction procedures result in pregnancy, and among these pregnancies, not all result in the birth of a child. In 2006, of the 99199 cycles of IVF carried out in human reproduction clinics in the United States, with the use of fresh semen, 34719 (35\%) led to a pregnancy, but only 28404 (29\%) resulted in a birth. In other words, $18 \%$ of the gestations conceived by IVF did not result in a birth (1).

Among the factors that influenced the results of IVF, semen quality parameters are accepted as a good indicator of the capacity for oocyte fertilization and attainment of pregnancy, along with the quality of the oocyte (4). The semen quality parameters recognized as vital for assessment of fertility are sperm concentration, motility and morphology. Various authors consistently correlate the values of sperm evaluation criteria with oocyte fertilization rates in IVF (5-7).

Sperm morphology is among the spermogram factors that displays a potential impact on male fertility. Among the various sperm morphology classification systems, the Tygerberg strict criterion, originally described by Kruger et al., in 1987 (8), which uses morphometric analysis to determine if the spermatozoon fits within a strict range considered normal, where normal is established as the value of more than $14 \%$ of the spermatozoa within the criteria established in a sample.

The currently recommended method for sperm morphology assessment is a classification of normal/abnormal spermatozoa, with an optional report of the site of abnormalities in abnormal sperm $(8,9)$. With the goal of performing this evaluation, the spermatozoa, which anatomically comprise head, neck, middle piece, main piece, and end piece, are divided only into head (and neck) and tail (middle piece and main piece). For a spermatozoon to be considered normal, both its head and tail must be normal. All the borderline forms should be considered abnormal (10).
Nevertheless, technical procedures for assessing sperm morphology are associated with a series of difficulties related to lack of objectivity, variation in interpretation, and poor performance in external evaluations of quality control (10).

In 1988, Kruger et al. demonstrated the importance of sperm morphology as a significant prognostic factor in IVF (11); currently however, strict morphology lacks reproducibility and clinical applicability as there is still doubt in more recent studies about the true implications of IVF results, particularly in reference to the classic technique (12).

Sperm morphology has been questioned by some authors about its real value as a prognostic factor for IVF, and most studies use the ICSI technique as standard for evaluation $(13,14)$. In a study performed in 2010, French et al. suggested that the prognostic value of strict morphology should be reconsidered in cycles of assisted reproduction involving IVF with ICSI, since the sperm morphology evaluated by Kruger strict morphology showed little prognostic value in IVF cycles with ICSI and did not seem to influence the development or the morphology of the blastocyst (15).

In studies carried out with the ICSI technique, the correlation between the two factors does not seem to exist, but when the technique used for IVF is the classic technique, the question remains about the true prognostic role of sperm morphology, even after three decades of experience with IVF $(12,15)$.

Considering latest data about sperm morphology, the World Health Organization changed the normal values for strict sperm morphology from $14 \%$ to $4 \%$ in order to fit the normality cutoff point to new evidence (16).

The objective of the present study is to determine the influence of the values of strict sperm morphology in men with normal sperm concentrations, according to the World Health Organization criteria, on the results of classic IVF.

\section{MATERIALS AND METHODS}

\section{Patients}

The individuals included in the study were selected from a review of clinical charts of couples 
who sought the assisted reproduction department of the Projeto Alfa clinic for pregnancy, during the years 2007 and 2010, due to conjugal infertility for at least 12 months.

This study included couples with female causes for infertility or couples with unexplained infertility at the clinical assesment. Prior participation in an IVF cycle or in any other assisted reproduction technique was not considered an exclusion factor for the study.

The male factor was assessed by means of a spermogram, and as an additional inclusion criterion, only men who presented with a spermogram within the range of normality for the parameters sperm concentration, sperm motility, volume of ejaculate, and total sperm count after semen processing by discontinuous gradient technique were included.

The following normality values were adopted: sperm concentration greater than 20 million per milliliter, sperm motility greater than 50\% of directional motility in the sample, volume of ejaculate greater than 1.5 milliliter, and total sperm count after semen processing greater than 20 million. All spermograms were evaluated by the same biologist using the manual technique.

This study was submitted to the Ethics and Research Committee of the Faculdade de Medicina do ABC and approved under Protocol \# 054/2010.

\section{Study design}

Based on the sperm morphology of the semen sample collected for the initial evaluation, patients were divided into three groups: in group A, patients with sperm morphology values between $0 \%$ and $4 \%$; in group B, between 5\% and 14\%, and in group $\mathrm{C}$, patients with sperm morphology greater than 14\%. Group C was considered the control group, and groups A and B were compared to the control group regarding parameters of female age, number of oocytes retrieved, number of embryos obtained, number of embryos transferred and male age.

The outcomes analyzed were oocyte fertilization rate, biochemical pregnancy rate, clinical pregnancy rate, and rate of liveborns. Comparison among the groups as to the outcomes was performed in a manner similar to that previously described for the other parameters.
The oocyte fertilization rate was defined by the number of zygotes with two pronucleii and two polar bodies when assessed between 16 and 18 hours after insemination, divided by the number of oocytes retrieved. Biochemical pregnancy was defined as patients with beta-HCG dosing greater than $20.0 \mathrm{mIU} / \mathrm{dL} 14$ days after the embryo transfer. Clinical pregnancy was defined as the presence of at least one gestational sac with fetal heartbeats on ultrasonography, one month after embryo transfer.

\section{Seminal Preparation and Evaluation of Sperm Morphology}

Semen samples were collected by masturbation two to five days after the last ejaculation, both for initial seminal analysis and for IVF. The initial seminal analysis of sperm concentration, sperm morphology and volume of ejaculate was performed according to the World Health Organization criteria (10).

Seminal processing was performed using the discontinuous gradient technique with the use of the culture medium Isolate (Irvine Scientific) and Spermgrad (Vitrolife), followed by evaluation of total seminal count in the sample retrieved.

Sperm morphology was evaluated in a single sample for the initial assessment of patients, counting 100 cells, according to the strict criteria proposed by Kruger/Tygerberg and adopted by the World Health Organization (10), using Papanicolaou stain.

\section{IVF Protocols}

Ovarian stimulation of multiple follicles was done with purified or recombinant gonadotrophines; GnRH agonists or antagonists were used to suppress endogenous secretion of gonadotrophines, with medication doses adjusted for each individual case, taking into consideration the woman's age and ancillary tests. Patients who presented with at least three follicles with at least 17 millimeters diameter on ultrasonography after ovarian stimulation received injections of hCG. Follicular puncture and aspiration was guided by transvaginal ultrasound and was performed 36 hours after the injection of hCG.

Gamete handling was carried out following routine protocols, with the classic IVF tech- 
nique using semen obtained by masturbation (17). Each oocyte was inseminated with approximately 200.000 sperm cells.

\section{Statistical Analysis}

Groups A and B were individually compared to group $\mathrm{C}$, used as the control group due to better sperm morphology. The variables of female age, male age, number of oocytes retrieved, number of embryos transferred, number of embryos obtained, and oocyte fertilization rate were analyzed using Student's two-tailed t test. The variables biochemical pregnancy rate, clinical pregnancy rate, and rate of liveborns were evaluated using the two-tailed chi-squared test. In both evaluations, a 5\% level of significance was adopted, with $80 \%$ statistical power.

Additionaly, the same statistical analysis above described was performed to compare group A to groups B and C together, according to the new World Health Organization criteria (16).

The sample size calculated to obtain a statistical power of $80 \%$ and a significance level of $5 \%$ for the variable clinical pregnancy was 27 cases in each group, based on analyses of the data from studies on the topic (18). The fertilization rate variable needs a smaller sample size in order to obtain the same statistical power with only 19 cases in each group (11).

\section{RESULTS}

\section{Patients}

Analysis was made of 1721 clinical charts of couples who underwent IVF cycles during the stated period, in which 244 met the inclusion criteria for this study. Based on review of the clinical charts, the three proposed groups were formed; group A included 27 cases; group B included 165 cases, and group $\mathrm{C}$ included 52 cases.

Among the causes of infertility that justified the use of IVF, the most common was the tubal factor with 119 cases, representing $48.8 \%$ of the total (Figure-1).

Groups A and B were compared isolatedly to group $C$ in an initial evaluation as to the variables female age, male age, number of oocytes retrieved in metaphase II (MII), number of embryos obtained and number of embryos transferred, and no statistical differences were found in any parameter among the groups (Table-1).

The overall mean of all 244 cases for female age were $34.5 \pm 4.26$ years; for male age it was $35.8 \pm 5.44$ years; for the number of oocytes retrieved in metaphase II it was $6.57 \pm 3.84$ oo-

Figure 1 - Proporcion of causes that led to IVF.

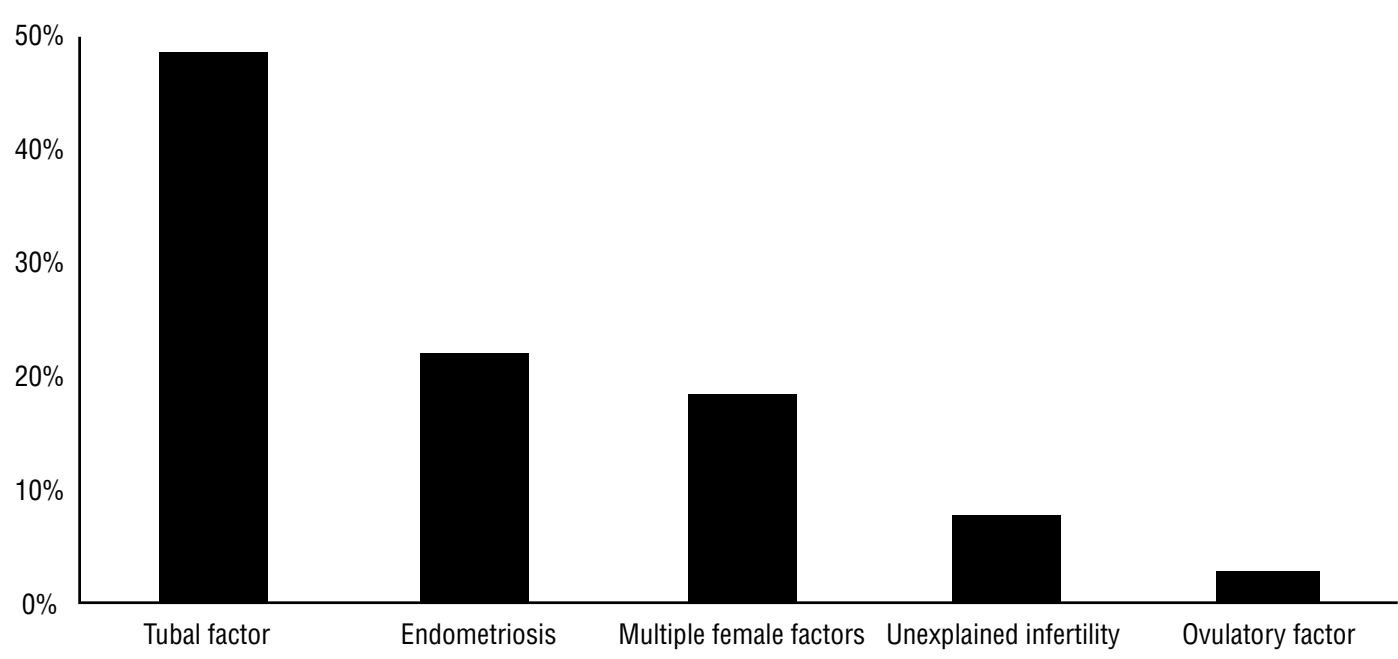


Table 1 - Means of groups and p value for statistical comparison of groups A and B with group C.

\begin{tabular}{lccccc}
\hline & Group A & Group B & Group C & p1* & p2** \\
\hline Female age & $34.1 \pm 4.1$ & $34.5 \pm 4.3$ & $34.6 \pm 4.1$ & 0.62 & 0.92 \\
Male age & $36.4 \pm 6.6$ & $35.7 \pm 5.4$ & $35.9 \pm 4.9$ & 0.72 & 0.82 \\
Number of MII oocytes retrieved & $6.00 \pm 3.0$ & $6.85 \pm 4.1$ & $5.99 \pm 3.1$ & 0,97 & 0.11 \\
Number of embryos obtained & $3.11 \pm 1.7$ & $4.13 \pm 2.2$ & $3.55 \pm 1.7$ & 0.29 & 0.15 \\
Number of embryos transferred & $2.40 \pm 1.0$ & $2.85 \pm 0.9$ & $2.73 \pm 0.9$ & 0.18 & 0.72 \\
\hline${ }^{*}$ p1 = group A x C & & & & & \\
** p2 = group B x C & & & & &
\end{tabular}

cytes; for the number de embryos obtained it was $3.80 \pm 2.11$ embryos; and for the number of embryos transferred it was $2.77 \pm 0.96$ embryos.

\section{IVF Results}

the results of IVF were compared in a similar manner to the parameters previously described in Table-1. In the variables analyzed: oocyte fertilization rate, biochemical pregnancy rate, clinical pregnancy rate, and rate of liveborn, no statistical difference was seen among the groups (Table-2).

The overall mean of the 244 cases for oocyte fertilization rate was $79.4 \% \pm 22.1 \%$; biochemical pregnancy rate was $37.2 \%$, clinical pregnancy rate was $27.8 \%$, and the rate of liveborns was $26.2 \%$.
The distribution of the total number of cases in each group and the respective number of gestations are represented in the graphic below (Figure-2). The mean oocyte fertilization rates are shown for each group (Figure-3).

Comparing group A to groups B and C together, no statistical difference on the variables female age, male age, number of oocytes retrieved in metaphase II (MII), number of embryos obtained and number of embryos transferred was found. Similarly to the analysis performed to groups $\mathrm{A}, \mathrm{B}$ and $\mathrm{C}$ isolated, the comparison between group $\mathrm{A}$ to groups $\mathrm{B}$ and $\mathrm{C}$ together showed no statistical difference in the variables of IVF results (Table-3).

Table 2 - Fertilization and pregnancy rates in the groups and p value for statistical comparison of groups A and B to group C.

\begin{tabular}{|c|c|c|c|c|c|}
\hline & Group A & Group B & Group C & $\mathrm{p} 1^{*}$ & $\mathrm{p} 2^{* *}$ \\
\hline Fertilization rate (Mean) & $71.9 \%$ & $80.9 \%$ & $78.8 \%$ & 0.21 & 0.51 \\
\hline Biochemical pregnancy rate & $44.4 \%$ & $33.3 \%$ & $36.5 \%$ & 0.49 & 0.97 \\
\hline Clinical pregnancy rate & $37.0 \%$ & $25.4 \%$ & $30.8 \%$ & 0.57 & 0.45 \\
\hline Liveborn rate & $33.3 \%$ & $24.2 \%$ & $28.8 \%$ & 0.68 & 0.50 \\
\hline
\end{tabular}


Figure 2 - Number of cases and number of cases with clinical pregnancy in the groups presented.

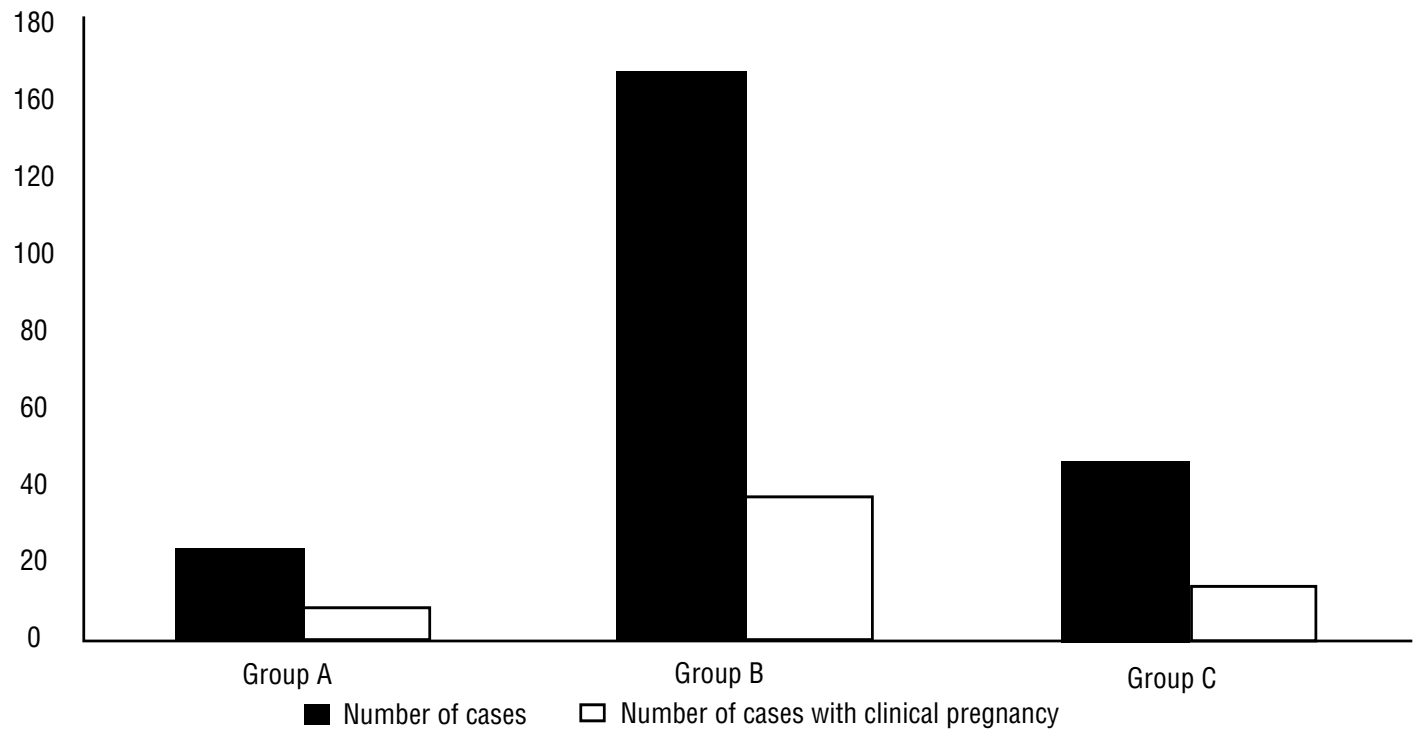

Figure 3 - Oocyte fertilization rate in the groups presented.

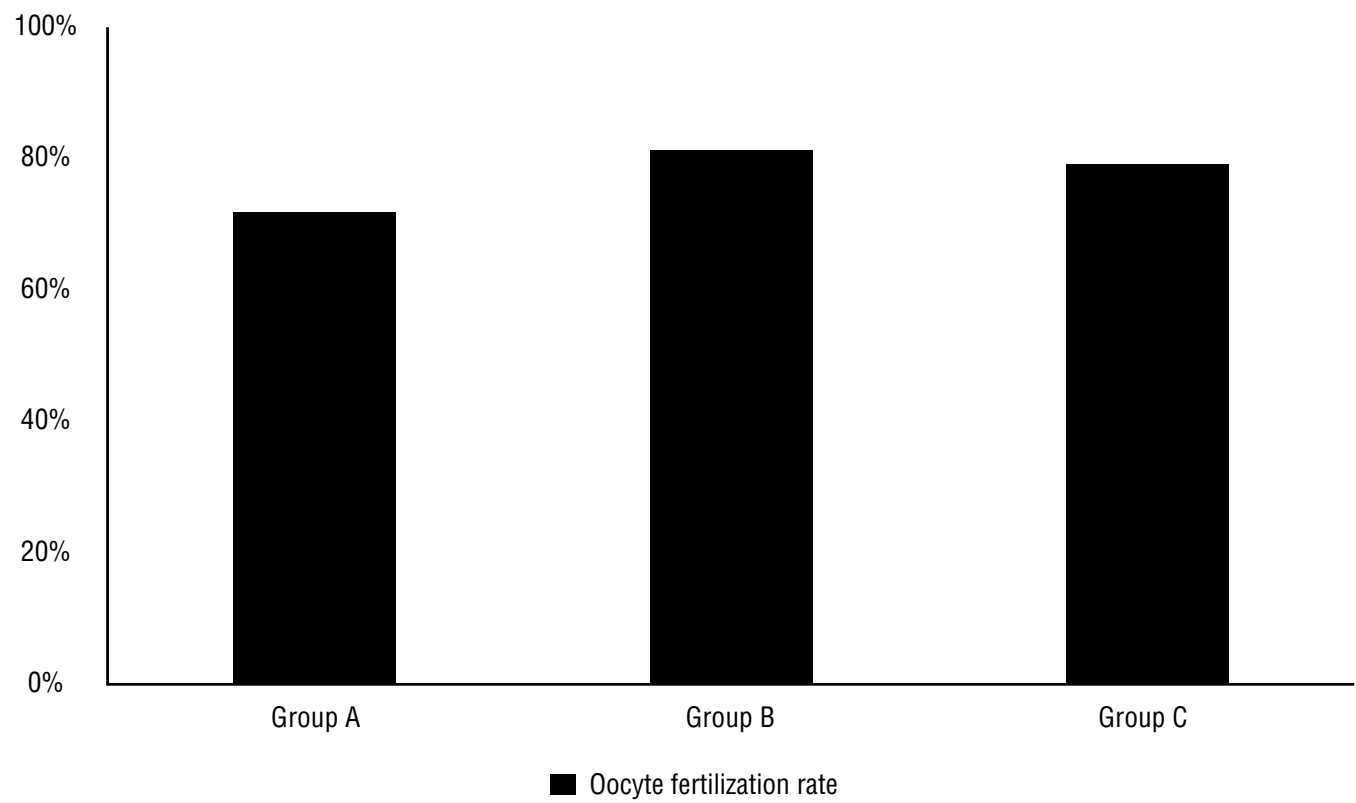


Table 3 - Fertilization and pregnancy rates in the groups and p value for statistical comparison of group A to groups B and C together.

\begin{tabular}{lccc}
\hline & Group A & Group B + C & p \\
\hline Fertilization rate (Mean) & $71.9 \%$ & $80.4 \%$ & 0.30 \\
Biochemical pregnancy rate & $44.4 \%$ & $34.1 \%$ & 0.29 \\
Clinical pregnancy rate & $37.0 \%$ & $26.7 \%$ & 0.26 \\
Liveborn rate & $33.3 \%$ & $25.3 \%$ & 0.37 \\
\hline
\end{tabular}

\section{DISCUSSION}

The present study is one of the few performed correlating sperm morphology exclusively with classic technique IVF cycles $(4,6,11,18-$ 20), not including cycles carried out using the ICSI technique. Its results demonstrated that the values of sperm morphology showed no correlation with pregnancy rates in IVF cycles.

Another positive characteristic of this study was sperm morphology evaluation as an isolated variable in spermogram, since the other seminal parameters were within normal values. Some similar studies performed previously showed other spermogram variables, such as sperm concentration or motility, variables that may have an influence on the results of IVF regardless of the values of sperm morphology (13).

The values of sperm morphology proposed to form the groups in this study were based on prior studies demonstrating that men with spermograms showing sperm morphology values of $14 \%$ or more would produce better results for IVF. In this way, the group with the morphology of $14 \%$ or more was established as the control group for comparison to the other two groups, which were divided using the cut-off value of $4 \%$ proposed in prior studies $(8,9,11)$.

The sperm morphology cut-off values also follow the new classification proposed by the World Health Organization. The sperm morphology assessment proposed in World Helth Organization's latest manual intends to limit what is identified as normal to the potentially fertilizing spermatozoa. Using these guidelines, the range of percentage normal values for both fertile and infertile men is likely to be $0-30 \%$, with few samples exceeding 25\% of normal spermatozoa. This low value will inevitably produce low thresholds; indeed reference limits and thresholds of 3-5\% normal forms (16).

The new normality cut-off point proposed in the referred manual is $4 \%$ and this information led to another statistical analysis between the group A and the groups B and C together representing the samples considered as morphologically normal.

The three groups formed showed no statistical differences when compared to possible confounding factors, such as female age, number of oocytes retrieved, number of embryos obtained, and the number of embryos transferred, allowing sperm morphology to remain as the only spermogram variable among the groups, which proved to be comparable.

Other factors with possible influence on the results were not statistically analyzed, such as the cause for female infertility that indicated IVF and the medication used for ovarian stimulation as well as its doses, due to the high number of variables within each factor, which would not allow sufficient numbers in each group for adequate statistical analysis.

One potential factor of influence in the study results was that the morphology reading was performed on a spermogram prior to the performance of IVF and not on the sample used for the procedure itself. Even considering that the 
time elapsed between the sample for the reading and the performance of IVF was no more than two months, changes in the values of morphology between the two readings could influence the study results. Nevertheless, the authors did not consider this factor relevant, in general, to the sample.

Keegan et al. carried out a study with 495 infertile couples in which men presented with spermograms containing more than two million mobile spermatozoa after seminal processing, and compared patients with strict morphology greater than or equal to five with patients who had strict morphology smaller than five using classic IVF and ICSI cycles; no statistical difference was noted between the oocyte fertilization rates and liveborn rates with either one of the techniques (12). In a similar study, French et al. analyzed 1074 IVF cycles done exclusively with the ICSI technique, and compared the results of oocyte fertilization rates and rates of liveborns in patients with strict morphology values that varied between zero and seven, without finding any statistical difference between the groups, however in this study the patients with other altered spermogram parameters were not excluded from the study (15).

The results of the present study go against those reached in primary studies on the topic $(4,8,9,11,21)$. Among these studies, one of the most significant is the one by Kruger et al., who first compared results of oocyte fertilization rates and pregnancy rates after IVF cycles in 96 couples, in which men presented with a spermogram with strict morphology greater than or equal to $14 \%$ and other seminal parameters within normality, noting a statistical difference in the given variables in favor of the group with strict morphology greater than 14\% (8).

One possible explanation for the result herein presented having been different from initial studies correlating sperm morphology and IVF results may be attributed to the sperm morphology assessment technique used, hence the significant variability and inconsistency in the results when comparing different institutions, different professionals, and even among observations made by the same professional (22).
In this study, the spermograms were all interpreted by the same professional with the intention of decreasing error and variability noted among different professionals and following the criteria for morphological sperm evaluation adopted by the World Health Organization (10) in the strictest way possible.

The prognostic evaluation for IVF founded on spermogram parameters is based on sperm concentration and motility and lacks instruments for sperm morphology evaluation with precise and reproducible results. Perhaps new morphological classifications are needed to improve accuracy of the evaluation.

One of the techniques proposed for morphological sperm assessment is the motile sperm organelle morphology examination (MSOME), which evaluates the sperm under the microscope with a magnification of at least 1000 times; this technique positively correlated sperm nuclear morphology with oocyte fertilization and pregnancy rates, although experience with this technique is still too limited to enable definitive conclusions on the topic (23).

Use of the electronic microscope to evaluate ultrastructural spermatic morphology of the components of the sperm head was used in a study by Mashiasch et al., in 1992, and correlated with the capacity for fertilization of the sperm in in vitro assessments (7).

Other forms of evaluating the sperm to determine the prognosis of IVF have been proposed, basing the tests on the functional evaluation of the sperm, such as, for example, acrosome reaction test and hemizona assay, although these tests still lack clinical applicability, and despite promising results presented, remain as tools for the experimental laboratory.

The conclusion of this study was that the values of strict sperm morphology, as proposed by Kruger and adopted by the World Health Organization, had no influence on the results of classic in vitro fertilization in the sample studied.

\section{CONFLICT OF INTEREST}

None declared. 


\section{REFERENCES}

1. Sunderam S, Chang J, Flowers L, Kulkarni A, Sentelle G, Jeng $G$, et al.: Assisted reproductive technology surveillance--United States, 2006. MMWR Surveill Summ. 2009; 58: 1-25.

2. ESHRE Capri Workshop Group: Intrauterine insemination. Hum Reprod Update. 2009; 15: 265-77.

3. Palermo G, Joris H, Devroey P, Van Steirteghem AC: Pregnancies after intracytoplasmic injection of single spermatozoon into an 00cyte. Lancet. 1992; 340: 17-8.

4. Kruger TF, Menkveld R, Stander FS, Lombard CJ, Van der Merwe JP, van Zyl JA, et al.: Sperm morphologic features as a prognostic factor in in vitro fertilization. Fertil Steril. 1986; 46: 1118-23.

5. Oehninger $\mathrm{S}$, Kruger T: The diagnosis of male infertility by semen quality. Clinical significance of sperm morphology assessment. Hum Reprod. 1995; 10: 1037-8.

6. Enginsu ME, Dumoulin JC, Pieters MH, Evers JL, Geraedts JP: Predictive value of morphologically normal sperm concentration in the medium for in-vitro fertilization. Int $\mathrm{J}$ Androl. 1993; 16: 113-20.

7. Mashiach R, Fisch B, Eltes F, Tadir Y, Ovadia J, Bartoov B: The relationship between sperm ultrastructural features and fertilizing capacity in vitro. Fertil Steril. 1992; 57: 1052-7.

8. Kruger TF, Acosta AA, Simmons KF, Swanson RJ, Matta JF, Veeck LL, et al.: New method of evaluating sperm morphology with predictive value for human in vitro fertilization. Urology. 1987; 30: 248-51.

9. Coetzee K, Kruge TF, Lombard CJ: Predictive value of normal sperm morphology: a structured literature review. Hum Reprod Update. 1998; 4: 73-82.

10. World Health Organization. WHO laboratory manual for the examination and processing of human semen. 4th ed. Geneva: World Health Organization. 1999.

11. Kruger TF, Acosta AA, Simmons KF, Swanson RJ, Matta JF, Oehninger S: Predictive value of abnormal sperm morphology in in vitro fertilization. Fertil Steril. 1988; 49: 112-7.

12. Keegan BR, Barton S, Sanchez X, Berkeley AS, Krey LC, Grifo $\mathrm{J}$ : Isolated teratozoospermia does not affect in vitro fertilization outcome and is not an indication for intracytoplasmic sperm injection. Fertil Steril. 2007; 88: 1583-8.

13. McKenzie LJ, Kovanci E, Amato P, Cisneros P, Lamb D, Carson SA: Pregnancy outcome of in vitro fertilization/intracytoplasmic sperm injection with profound teratospermia. Fertil Steril. 2004; 82: 847-9.
14. Høst E, Ernst E, Lindenberg S, Smidt-Jensen S: Morphology of spermatozoa used in IVF and ICSI from oligozoospermic men. Reprod Biomed Online. 2001; 3: 212-5.

15. French DB, Sabanegh ES Jr, Goldfarb J, Desai N: Does severe teratozoospermia affect blastocyst formation, live birth rate, and other clinical outcome parameters in ICSI cycles? Fertil Steril. 2010; 93: 1097-103.

16. World Health Organization. WHO laboratory manual for the examination and processing of human semen. 5 th ed. Geneva: World Health Organization. 2010.

17. Gianaroli L, Plachot M, van Kooij R, Al-Hasani S, Dawson $\mathrm{K}$, DeVos $A$, et al.: ESHRE guidelines for good practice in IVF laboratories. Committee of the Special Interest Group on Embryology of the European Society of Human Reproduction and Embryology. Hum Reprod. 2000; 15: 2241-6.

18. Ombelet W, Fourie FL, Vandeput $H$, Bosmans E, Cox A, Janssen M, Kruger T: Teratozoospermia and in-vitro fertilization: a randomized prospective study. Hum Reprod. 1994; 9: 1479-84.

19. Grow DR, Oehninger S, Seltman HJ, Toner JP, Swanson RJ, Kruger TF, et al.: Sperm morphology as diagnosed by strict criteria: probing the impact of teratozoospermia on fertilization rate and pregnancy outcome in a large in vitro fertilization population. Fertil Steril. 1994; 62: 559-67.

20. Robinson JN, Lockwood GM, Dokras A, Egan DM, Nicholson SC, Ross C, et al.: Does isolated teratozoospermia affect performance in in-vitro fertilization and embryo transfer? Hum Reprod. 1994; 9: 870-4.

21. Kobayashi T, Jinno M, Sugimura K, Nozawa S, Sugiyama T, lida E: Sperm morphological assessment based on strict criteria and in-vitro fertilization outcome. Hum Reprod. 1991; 6: 983-6.

22. Ombelet W, Bosmans E, Janssen M, Cox A, Maes M, Punjabi $U$, et al.: Multicenter study on reproducibility of sperm morphology assessments. Arch Androl. 1998; 41: 103-14.

23. Bartoov B, Berkovitz A, Eltes F, Kogosowski A, Menezo Y, Barak $Y$.: Real-time fine morphology of motile human sperm cells is associated with IVF-ICSI outcome. J Androl. 2002; 23: 1-8.

Correspondence address: Dr. Milton Ghirelli-Filho Address: Rua das Laranjeiras, 369 / 54 Santo André, 09070-560, SP, Brazil Fax: + 5511 4994-9611 E-mail: nghirelli@uol.com.br 


\section{EDITORIAL COMIMENT}

This is an interesting retrospective series demonstrating the lack of influence of isolated low morphology values in biochemical and clinical pregnancy rates following classic IVF treatment. Care was taken only to include couples with an unknown or female factor cause of infertility.

Assessing morphology is not a very precise laboratory task as it may vary according to subjective biologist evaluation and even from same patient's sample collection at different oc-

\section{REFERENCES}

1. Esteves SC, Zini A, Aziz N, Alvarez JG, Sabanegh ES Jr, Agarwal A: Critical appraisal of World Health Organization's new reference values for human semen characteristics and effect on diagnosis and treatment of subfertile men. Urology. 2012; 79: 16-22. casions. Use of semen reference values provided by 2010 WHO latest publication must also be looked at with care (1).

This paper conclusion makes us wonder if there has been overtreatment of isolate teratozoospermia patients with IVF/ ICSI as pregnancy rates seem equivalent throughout low and higher values. Prospective studies might help answering this question as would analysis of low morphology-associated functional variables such as sperm DNA integrity and chromatin condensation (2).

2. Abu Hassan Abu D, Franken DR, Hoffman B, Henkel R: Accurate sperm morphology assessment predicts sperm function. Andrologia. 2012; 44(Suppl 1): 571-7.

Dr. Ricardo Miyaoka

Department of Urology

University of Campinas, Unicamp, Brazil Cidade Universitária "Zeferino Vaz"

Barão Geraldo - Campinas, São Paulo, 13083-970, Brasil E-mail:rmiyaoka@uol.com.br 